\title{
Dehiscencia del canal semicircular superior detectada durante un tratamiento dental
}

\section{Superior semicircular canal dehiscence detected during dental treatment}

\section{Crovetto Martínez R*, Martínez Rodríguez A**, Cisneros Gimeno AI***, Gracia Tello B****}

\section{RESUMEN}

La dehiscencia de canal semicircular superior del oído interno se caracteriza por la falta de cobertura ósea en la parte superior de dicho canal. Los pacientes con esta enfermedad pueden presentar vértigo cuando son sometidos a sonidos intensos, lo que se conoce como fenómeno de Tullio. Presentamos un caso clínico que asoció dicho fenómeno al fresado de un molar inferior, siendo este el primer síntoma de su enfermedad.

Palabras clave: Dehiscencia, canal semicircular superior, fenómeno de Tullio tratamiento dental.

\section{SUMMARY}

Superior Semicircular Canal dehiscence is characterized by an osseous defect in its upper aspect. Patients with this condition may have sound-induced vertigo (Tullio phenomenon). We report a case in which a patient had a Tullio phenomenon in response to the drill of a lower molar, which is the first symptom disease.

Key words: Dehiscence; superior semicircular canal, Tullio phenomenon, dental treatment.

Fecha de recepción: 13 de junio de 2013.

Aceptado para publicación: 4 de julio de 2013.

* Profesor Asociado de Odontología de la UPV/EHU. Licenciado en Odontología. Doctor por la UPV/EHU.

** $\quad$ Profesor Titular de la UPV/EHU.

*** Profesor Contratado Doctor. Facultad de Medicina. Universidad de Zaragoza.

**** Médico Residente. HCU Lozano Blesa. Zaragoza.

Crovetto Martínez R, Martínez Rodríguez A, Cisneros Gimeno AI, Gracia Tello B. Dehiscencia del canal semicircular superior detectada durante un tratamiento dental. Av. Odontoestomatol 2013; 29 (4): 207-210.

\section{INTRODUCCIÓN}

En condiciones normales, el oído interno tiene dos ventanas, la oval y la redonda y ello condiciona su fisiología (Figura 1). La dehiscencia del canal semicircular superior (DCSS) es una alteración del oído interno caracterizada por la ausencia de parte de la cobertura ósea de dicho canal, determinando la pre- sencia de tres ventanas en el oído interno, la oval, la redonda y la dehiscente (Figura 2).

Clínicamente la DCSS puede provocar alteraciones auditivas tales como oír con eco su propia voz (autofonía) o presentar hipoacusia. Otra de las manifestaciones clínicas propias de la DCSS es el vértigo, que suele ser causado por los cambios de presión en el 


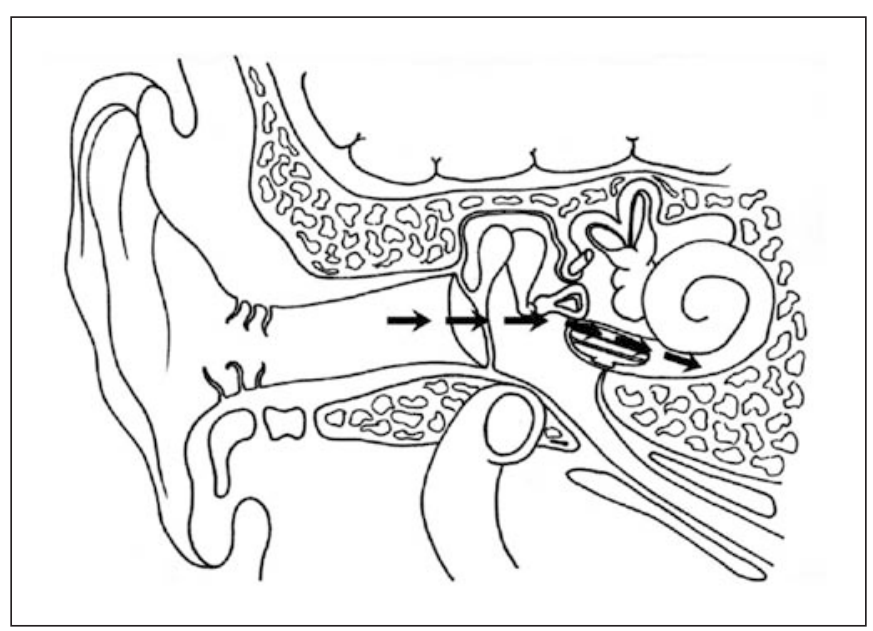

Fig. 1. Esquema del oído normal. Las flechas muestran el recorrido fisiológico de la energía sonora que alcanza el oído interno. Se observa que la energía sonora se dirige exclusivamente hacia el caracol, determinando sensaciones exclusivamente auditivas.

oído producidos al sonarse, toser, estornudar o hacer esfuerzos o al hacer la maniobra de Valsalva. Otra manifestación clínica de la DCSS, especialmente importante para el Odontólogo, es el desencadenamiento del vértigo en respuesta a estímulos sonoros de elevada intensidad, lo que se conoce como fenómeno de Tullio (1).

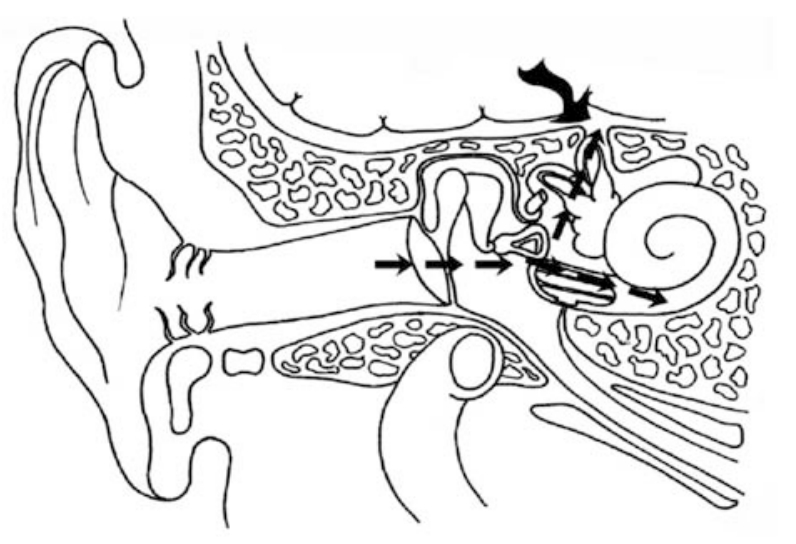

Fig. 2. Este esquema del oído interno muestra una dehiscencia en el techo del canal semicircular superior que hace el papel de "tercera ventana" (flecha irregular). Las flechas rectas muestran el recorrido de la energía sonora que alcanza el oído. Parte de la misma se dirige en la dirección normal hacia el caracol auditivo pero la otra se dirige anormalmente hacia la "tercera ventana", estimulando las terminaciones del equilibrio y causando vértigo.
En este artículo presentamos un caso de DCSS cuyo primer síntoma vestibular tuvo lugar en el gabinete odontológico, durante la realización de una obturación dental.

\section{CASO CLÍNICO}

Mujer de 57 años que acudió al gabinete odontológico para realizar el tratamiento de una caries en la cara oclusal del $2^{\circ}$ molar inferior derecho (47) Con la paciente semitumbada, sin extensión cervical, se realizó la infiltración de anestesia local sin que se presentaran reacciones anómalas. Posteriormente se procedió a realizar un fresado de la caries con la turbina. Durante el fresado la paciente presentó un vértigo rotatorio. El vértigo cedió en el mismo momento en que se de detuvo el trabajo con la turbina. Reiniciado el fresado la paciente volvió a presentar vértigo rotatorio, mas leve en esta ocasión lo que permitió terminar la obturación sin problemas añadidos. La paciente fue remitida al Otorrinolaringólogo para estudio de su vértigo en respuesta al sonido intenso (fenómeno de Tullio). La anamnesis, las pruebas audiológicas (audiometría e impedanciometría), vestibulares (registro de nistagmus vértico-rotatorio frente a estímulos sonoros de elevada intensidad), neurofisiológicos (registro de potenciales vestibulares miogénicos evocados anormales) y la tomografía computarizada demostraron la existencia de una dehiscencia del canal semicircular superior derecho.

\section{DISCUSIÓN}

La DCSS fue descrita en 1998 por Minor (1). En condiciones normales el oído interno solamente tiene dos ventanas, la ventana oval y la redonda, ambas cerradas elásticamente. En estas condiciones los estímulos sonoros solo alcanzan el caracol del oído interno, induciendo respuestas auditivas (Figura 1). Sin embargo, cuando hay una DCSS existe una "tercera ventana" en el laberinto, lo que cambia la fisiología del oído interno. Así, los sonidos que alcanzan el oído interno no se dirigen solamente hacia el caracol auditivo como debieran, sino que se dirigen también a la "tercera ventana" situada en el canal semicircular superior implicado en el control del equilibrio (Figura 2) (2,3). Ello tiene dos consecuen- 
cias, la primera es que la energía sonora que alcanza el caracol auditivo es menor de la que debiera lo que provoca hipoacusia. La segunda consecuencia es que la energía sonora se transmite hacia el canal semicircular superior provocando la estimulación anormal de las terminaciones sensoriales del mismo causando vértigo y nistagmus, lo que se conoce como fenómeno de Tullio $(3,4)$.

En 1928, Tullio (5) observó respuestas vestibulares en conejos sometidos a estímulos sonoros de muy alta intensidad y concluyó sus hallazgos al encontrar iguales respuestas con estímulos sonoros de menor intensidad siempre que realizara una fístula experimental en los canales semicirculares de los animales. Desde la descripción de estos hallazgos se denomina fenómeno de Tullio al desencadenamiento de síntomas vestibulares (vértigo y nistagmo) en respuesta a la estimulación sonora. La intensidad sonora necesaria para despertar un fenómeno de Tullio en un sujeto con DCSS es de 90-100 dB con frecuencias de 250 a $4 \mathrm{Khz}(6,7)$. La intensidad del sonido generado por una turbina durante el fresado dental es variable en función de los equipos utilizados. La intensidad sonora generada durante el periodo de corte es de 65 y $85 \mathrm{~dB}$ SPL (sound pressure level), medida a 15-25 cm de la pieza de mano, siendo la turbina de alta velocidad la que genera intensidades sonoras mayores durante el fresado (8-11) lo que no parece suponer un riesgo mayor para el oído del dentista. Sin embargo, el sonido alcanza el oído interno del paciente por vía ósea con toda la intensidad que la turbina genera ya que el sonido se transmite por medios sólidos, como es el cráneo, con enorme eficacia y máxima velocidad (12). Ese estímulo acústico es esporádico y ocasional por lo que no hay problemas mayores en el oído del paciente sano. Sin embargo, cuando el sonido provocado durante el fresado alcanza el oído de un paciente con DCSS se produce un fenómeno de Tullio, ya que la intensidad sonora que alcanza su oído interno supera los $95 \mathrm{~dB}$ SPL precisos para desencadenar un vértigo rotatorio. Esto es lo que ha sucedido en la paciente que presentamos, cuyo ulterior estudio de mostró la presencia de una DCSS. Esta es la segunda comunicación de dicho fenómeno en el curso de un tratamiento dental, la primera en lengua española, ya que Kaski et al (2012)(13) han publicado un hallazgo similar en lengua inglesa. Considerando que la DCSS fue reconocida en 1998 en USA y que su primera publicación en lengua española data del 2005 (14) es muy probable que en el futuro estas comunicaciones se hagan más frecuentes.

La DCSS no es la única causa de vértigo que presentan los pacientes en el gabinete dental, existiendo otras causas para el mismo, como el vértigo posicional paroxístico benigno (VPPB) pero en este caso el vértigo se relaciona con cambios posturales entre situación incorporada y decúbito (15) y no con el estímulo acústico relacionado con el fresado como sucede en la DCSS. Muchos pacientes que sufren estos cuadros vertiginosos son considerados equivocadamente como nerviosos o neuróticos, por lo que deben conocerse estos procesos lo bastante para evitar esos errores $(6,14)$.

\section{BIBLIOGRAFÍA}

1. Minor LB, Solomon D, Zinreich JS, Zee DS. Sound-and/or pressure-induced vertigo due to bone dehiscence of the superior semicircular canal. Arch Otolaryngol Head Neck Surg 1998; 124:249-58.

2. Minor LB. Clinical manifestations of superior semicircular canal dehiscence. Laryngoscope 2005; 115:1717-27.

3. Crovetto-de la Torre MA, Whyte-Orozco J, CrovettoMartínez R, Whyte-Orozco A, Obon-Nogues JA, Martínez-Rodríguez A. A new cause of structural vertigo: superior semicircular canal dehiscence Rev Neurol 2011 Jun;16;52(12):751-8.

4. Yagi T, Koizumi Y. 3D analysis of cough-induced nystagmus in a patient with superior semicircular canal dehiscence. Auris Nasus Larynx 2009; 36:590-3.

5. Tullio P. L’oreggio. Bologna: L. Capelli; 1928.

6. Minor LB. Superior canal dehiscence syndrome. Am J Otol 2000;21(1):9-19).

7. Halmagyi GM, Swee T, McGarvie LA, Todd MJ, Bradshaw A, Yavor RA et al. Superior semicircu- 
lar canal dehiscence simulating otosclerosis. J Laryngol Otol 2003 (July);117(7):553-7.

8. Man A, Neuman H, Assif D. Effect of turbine dental drill noise on dentist hearing. Isr $\mathrm{J}$ Med Sci 1982;18(4):475-7.

9. Letho T. Dentists' hearing and exposure to high speed turbine dental drill noise. Proc Finn Dent Soc. 1990;86(3-4):115-25.

10. Sorainen E, Rytkönen E. Noise level and ultrasound spectra during burring. Clin Oral Invest 2002;6(3):133-6.

11. Brusis T, Hilger R, Niggeloh R, Huedepohl J, Thiesen KW. Are professional dental health care workers (dentists, dental technicians, assistants) in danger of noise induced hearing loss? Laryngorhinootologie. 2008 May;87(5):335-40.

12. Stenfelt S. Acoustic and physiologic aspects of bone conduction hearing. Adv Otorhinolaryngol 2011;71:10-21.
13. Kaski D, Davies R, Luxon L, Bronstein M, Rudge P. The Tullio phenomenon: a neurologically neglected presentation. J Neurol 2012;259(1): 4-21.

14. Crovetto de la Torre MA, Whyte J, Cisneros AI, Basurko JM, Oleaga L, Sarrat R : Síndrome de dehiscencia del canal semicircular superior. Aspectos embriológicos y quirúrgicos. Acta Española Otorrinolaringol 2005;56:6-11.

15. Crovetto R, Martínez A. Vértigo posicional paroxístico benigno relacionado con los tratamientos dentales. Av Odontoestomatol 2012;28(4): 191-6.

\section{CORRESPONDENCIA}

Rafael Crovetto Martínez

Máximo Aguirre, 5, $4^{\circ} \mathrm{B}$

Getxo. Vizcaya. España

E-mail: macdlt@telefonica.net 